\title{
EXPLANATION OF PLATES
}

Figs. 6-10. Electron micrographs of cellulose formed by $A$. acetigenum

Plate 1

Fig. 1. Cellulose from ethylene glycol medium.

Fig. 2. Cellulose from glycerol medium.

Fig. 3. Cellulose from maltose medium.
Fig. 4. Cotton cellulose.

Fig. 5. Cellulose from glycerol medium. Rotation photograph.

\section{Plate 2}

Fig. 6. Cellulose formed in a glucose yeast-water medium and examined in an unwashed condition. Magnification, $\times 8400$. The majority of the threads are about $500 \mathrm{~A}$. in width.

Fig. 7. Washed material from a glucose yeast-water medium. Magnification, $\times 8000$. Threads mostly about $500 \mathrm{~A}$. wide with a minority measuring a little less than $250 \mathrm{~A}$. Two threads were found to be not greater than $200 \mathrm{~A}$. in width.
Fig. 8. Washed material from a medium consisting of a solution of glucose and inorganic salts. Magnification, $\times 9000$. Most of the threads have a width of approximately $500 \mathrm{~A}$., but one or two threads embedded in film are approximately $250 \mathrm{~A}$. wide.

Fig. 9. Preparation from a medium containing ethylene glycol and inorganic salts. Magnification, $\times 24,000$. This shows what appears to be amorphous matter with a very few fine threads, the narrowest of which has a width of less than $80 \mathrm{~A}$. Others measured less than $200 \mathrm{~A}$. and one band was found to be $250 \mathrm{~A}$. in width.

Plate 3

Fig. 10. Preparation from a medium containing glycerol and inorganic salts. Magnification, $\times 8500$. Masses of what apparently is amorphous material, together with a very few fine threads, can be seen. The narrowest thread has a width of $250 \mathrm{~A}$., others are $500 \mathrm{~A}$. wide, while yet other bands may be noted varying in width from 750 to $1000 \mathrm{~A}$.

Fig. 11. Sectional photograph of cellulose from a starch hydrolysate medium. Magnification, $\times 1600$.

\section{A Means of Metabolic Investigation of Small Portions of the Central Nervous System in an Active State}

\author{
By H. McILWAIN \\ Biochemical Laboratories, Institute of Psychiatry (British Postgraduate Medical Federation, \\ University of London), Maudsley Hospital, S.E. 5
}

\section{(Received 11 April 1951)}

A method is described below by which it is possible for the first time to study the metabolism of small isolated fragments of the central nervous system of higher animals, in a state of electrically induced activity. Increase in the metabolism of slices of cerebral cortex on electrical stimulation in vitro was reported recently (McIlwain, 1951 a). In the apparatus which was used then, direct contact was made throughout the experiment between the slice of tissue and two stimulating electrodes. The tissue weighed about 50-120 mg. It was realized that the scope of such investigations would be greatly increased if they could be applied to smaller fragments of tissue and without fixing them to electrodes. Regional differences in biochemical responses to stimulation, and in the action of drugs on such responses, could then easily be explored. It has now been found possible to study in this way fragments weighing a mg. or less.

\section{EXPERIMENTAL}

Guinea pigs and rats of various breeds and ages were used. They were killed by a blow on the neck or by decapitation, the skull opened and the brain removed. Tissue preparations were normally ready for placing in the thermostat (37 or $39^{\circ}$ ) 15-30 min. after death of the animal. Salines and means of electrical stimulation were those described previously (McIlwain, 1951 a). A commercial diathermy apparatus was also used. This depended on charging and discharging a condenser from a.c. mains, through a coil and spark gap. A secondary coil, adjustable in relation to the first, gave the impulses which were taken through a high-frequency meter 
to the vessels. The discharge consisted of extremely brief spikes (perhaps $0 \cdot 1 \mu \mathrm{sec}$.) of some millions/sec. in frequency.

Electrodes. The problems met in choosing materials for electrodes in the present vessels were similar to those discussed before (McIlwain, 1951 a). Again, silver was largely used for electrodes which were not required to be sealed through glass. Electrodes which were to be so sealed were made of platinum and, if they were to be in contact with the experimental saline, were plated with gold. Vessels with sealed gilded electrodes were cleaned in the usual way, except that chromic acid mixtures were not used for such vessels on the first few days after the mixtures had been prepared, but only after they had been in use for other cleaning for some days. This was because the impression was gained that traces of free chlorine were present initially but were gradually lost.
The connexions outside the vessel, from electrode to source of current, were made according to Fig. $3 a$ of McIlwain (1951 $a$ ) which involved a junction through mercury. It was found that great care was needed when filling and emptying the side arms carrying the mercury, in order to avoid spilling small droplets into the main vessel, as any contact between the mercury and gold immediately resulted in amalgama. tion. In place of the temporary rubber junctions, more permanent ones were therefore made with a cement or by sealing tungsten wires through the glass.

Design of cylindrical vessels: absorbing carbon dioxide in them. To allow a variety of electrode types to be examined, cylindrical vessels were used with large-sized joints $(C 24)$ connecting them to the manometers (Figs. 1 and 2). For the same reason the centre well for gas-absorbing reagents was omitted. These reagents were accommodated in the large
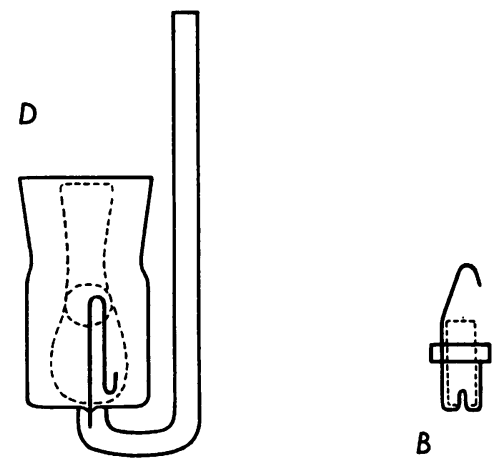

B
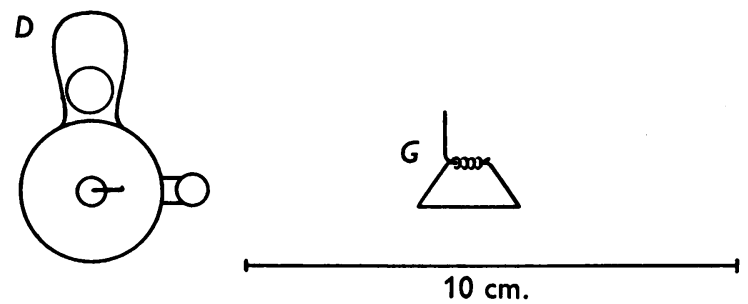

$A$
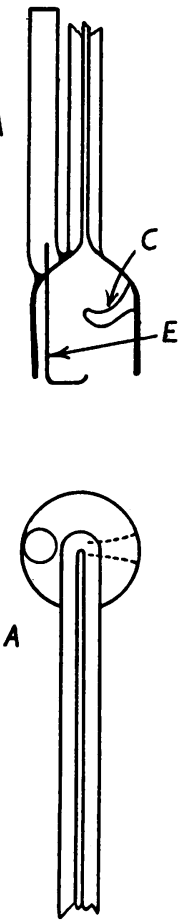

Fig. 1. Cylindrical electrode vessels and accessories. $D$, the vessels, with 1 side arm with valve stopper, and a wire electrode. The electrode would be plated in about the position shown and later bent to a shape such as $I$ of Fig. 2, or have attached to it other of the electrodes of Fig. 2. $A$, electrode manometer for vessel $D ; B$, pendant of silver wire (26 gauge) and polythene for holding a roll of filter paper, shown dotted, for absorbing agents; in use it is attached to the glass hook $C$. $G$, elevation of electrode $G$, Fig. 2, showing how it is attached to the electrode $E$ of the manometer.

The platinum wire, which was to form the basis of the electrodes in the conical and cylindrical vessels, was sealed to the vessels in slight excess of the amount estimated to be needed. It was then bent roughly to position and trimmed to a suitable length. It was then plated, and moved at intervals during plating, ensuring especially that no part of the wire was touching the vessel wall except at the point of the seal. It was then bent to its final position with small wooden sticks and with forceps which had small plastic sleeves slipped over them where they were to grip the wire. head which the manometer carried. Fig. $1 B$ shows the pendant devised for this purpose. At the beginning of an experiment a strip of hardened filter paper about $17 \times 35 \mathrm{~mm}$. was rolled and put in the polythene ring, so that it rested on the silver bridle. $5 \mathrm{~N}-\mathrm{NaOH}(0.15 \mathrm{ml}$.) was pipetted as a single drop into a small polythene weighing bottle, the paper in its pendant rested on the drop and the weighing bottle was closed so that atmospheric $\mathrm{CO}_{2}$ would not be absorbed. This was done $0.5 \mathrm{hr}$. or more before the absorbent was needed. After saline and tissue had been added to the 
manometric vessel and the manometer greased and connected to its oxygen supply, the pendant was taken from its bottle, held below by forceps, its upper loop hung over the glass hook on the manometer head, and the vessel put on the manometer.

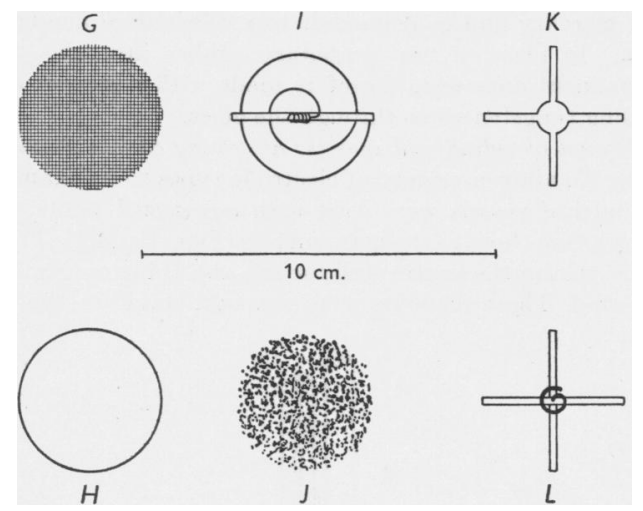

Fig. 2. Electrode patterns referred to in Table 1. $G$ (see also Fig. 1) was of silver gauze (wire $0.2 \mathrm{~mm}$. diam., mesh 18/cm.); $H$ and $K$ of silver sheet; $J$ formed on the base of the vessel by a silvering mixture (Johnson, Matthey and Co.); $I$ and $L$ of gold-plated platinum, if as lower electrodes fixed to the vessel, or of silver wire with the Perspex spacers shown, if hanging.

Fig. 3 gives the result of experiments which were carried out to see how effectively the alkali paper of the pendant absorbed $\mathrm{CO}_{2}$. It proved to be as effective as the paper in the normal centre wells of conical Warburg vessels of similar volume. The shaking rate in experiments with the cylindrical vessels was about $120 / \mathrm{min}$. This relatively high rate was found necessary in order to keep the tissue suspended.

Effect of passing current in the absence of metabolizing tissue. Various chemical and physical effects of passing currents through saline and substrate mixtures were noted in an earlier paper (McIlwain, $1951 a$ ). To avoid confusing any of these with a metabolic response of the tissue being investigated, each new electrode arrangement was examined in the following ways. (a) For gas change in the saline mixture without substrate; electrolysis by excessive currents or current densities could then be seen. (b) For gas change in the presence of substrate and any absorbing agents. This was carried out periodically in all vessels to ensure that the plating was intact. (c) For any change in a saline in which tissue had metabolized but had been removed. The effects of a labile metabolic product, not evident in $(b)$, would have been seen, but no instance of this was found.

Chopped tissues. These were prepared as follows. The tissue, for example the sheet of cortex obtained by unfolding a guinea pig hemisphere (McIlwain, 1951 a), was placed horizontally on a pad of several filter papers moistened with the experimental saline, and was first cut in one direction to strips 1-2 mm. wide with razor blading. Keeping the blade vertical, but in a direction approaching right angles to the first direction, the blade was then moved up and down rapidly while the tissue was moved under the blade, in the fashion adopted when chopping vegetables with a knife. The chopped tissue was washed from the filter paper into a small dish of saline with a wide-mouthed Pasteur pipette, and sucked up and down in the pipette a few times in order to separate clumps of fragments which were not completely cut. The tissue was transferred to experimental vessels by scooping it up on a small gauze shovel. This was made from a piece of silver gauze (mesh $18 / \mathrm{cm}$. wire $0.2 \mathrm{~mm}$. diam.) about $8 \times 12 \mathrm{~mm}$. mounted in a handle such as those used for bacteriological wire-loops. In this way, extremely small fragments of tissue were allowed to escape through the gauze. The amount scooped up for each vessel was judged

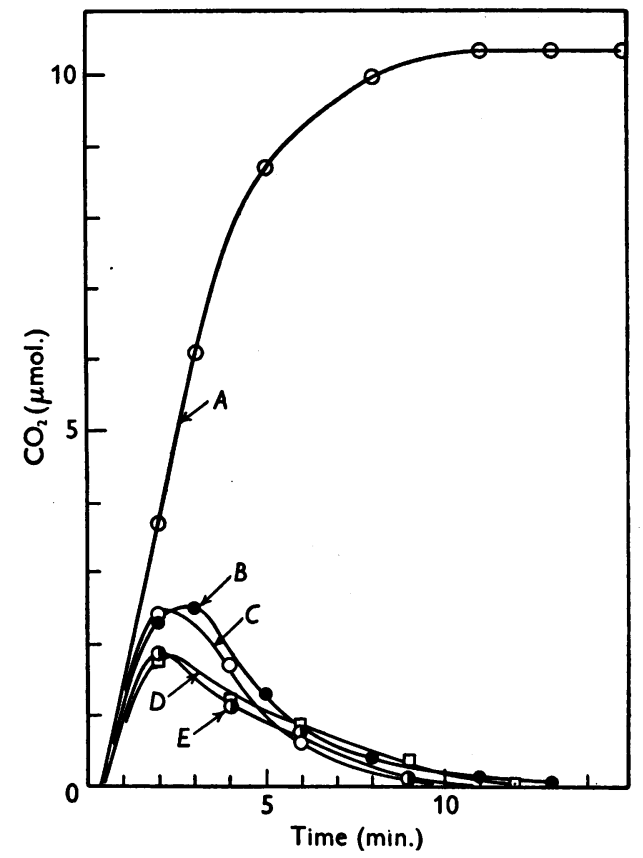

Fig. 3. Showing efficiency of $\mathrm{CO}_{2}$ absorption by alkali-paper in different vessels. Main compartments of the vessels contained $3 \mathrm{ml} .0 \cdot 1 \mathrm{~N}-\mathrm{H}_{2} \mathrm{SO}_{4}$ and the side arms, $0.5 \mathrm{ml}$. $0.02 \mathrm{M}-\mathrm{Na}_{2} \mathrm{CO}_{3}$. $A$, no absorbing reagents; $B-E$, with filter paper and $5 \mathrm{~N}-\mathrm{NaOH}$. $B$ and $C$, Warburg vessels of about $18 \mathrm{ml}$. and $D$ and $E$, cylindrical vessels of $23 \mathrm{ml}$.

visually to be 60-70 mg., when working at $20 \mathrm{mg} . / \mathrm{ml}$. and with vessels taking $3-3.5 \mathrm{ml}$; after practice this amount could be judged by its appearance on the scoop or in the vessel. At the end of the experiment the contents of each vessel were filtered through asbestos in a Gooch crucible. If needed for analysis, a specimen of the filtrate was then taken and the vessel washed out with $0.9 \% \mathrm{NaCl}$. The crucible, which had previously been prepared by washing, drying and weighing in the same way, was also washed with $0.9 \% \mathrm{NaCl}$, dried at $100^{\circ}$ and weighed. Wet weight of cerebral cortex was calculated from the dry weight by the factor $7 \cdot 1$ (McIlwain, $1951 a$ ).

Homogenates were prepared in all-glass test-tube homogenizers, from 1 part of cerebral cortex and 3 parts of glucosephosphate saline cooled in ice. The instrument was driven rapidly and homogenizing took 20 sec.; the suspension was passed through a wisp of glass wool in a small funnel and pipetted to the experimental vessels. Another portion was taken for dry weight. For comparison, wet weights were calculated as above. 


\section{RESULTS \\ Stimulation of slices without fixing them to electrodes}

Tissue fixed to a variety of electrodes could be stimulated in salines by impulses which were without action on slices floating freely in salines containing similar electrodes (McIlwain, 1951 $a$ ). However, these experiments were arranged so that the free slices did not come between the electrodes, where they would have been in danger of being entangled in them. They were commonly a cm. or so away, and not in the immediate field produced by the applied impulses. Moreover, a stimulus could be effective when only one pole was directly connected to the tissue (Fig. 8 of McIlwain, 1951 $a$ ). A factor conditioning stimulation was likely to be the potential gradient to which the tissue was exposed. Stimulation could be brought about by peak voltages of 3-5 V. between electrodes 2-10 mm. apart. It seemed possible by suitable electrode arrangements to include most of the fluid in an experimental vessel in such a field.

In devising the conditions under which this might be done, vessels $D$, Fig. 1, have largely been used. They and the corresponding manometers, and their handling, are described in the experimental part. The vessels were made cylindrical so that the variety of electrode types of Fig. 2 could be examined. One of these was normally at the bottom of a column of saline about $22 \mathrm{~mm}$. in diameter and $7 \mathrm{~mm}$. deep. The other was parallel to the first and about $5 \mathrm{~mm}$. above, so that it was near the surface of the saline. The tissue was between the two, and by rapid shaking (about 120 complete oscillations of $8 \mathrm{~cm} . / \mathrm{min}$.) was maintained in suspension in the fluid.

Impulses of characteristics similar to those effective when applied to tissue-holding electrodes were not always effective when applied to the electrodes of vessels $D$ (Table 1; Fig. 1). Alternating current was not suitable for stimulation owing to the relatively large amount of energy expended. Thus with electrode $G$ above and $H$ below, application of $2 \mathrm{~V}$. at 50 cyc./sec. caused a pressure change of some $25 \mathrm{~mm}$. corresponding to a mean temperature increase of about $0 \cdot 8^{\circ}$. No increase in respiration resulted (Fig. $4 B$ ). Use of electrodes $J$ or $K$ below, retaining $G$ above, was no more satisfactory. In attempts to apply greater potential gradients across the vessel condenser pulses were used, as these could give a much higher peak voltage with a given amount of energy. Pulses at $24 \mathrm{~V}$. and reasonable capacities and frequencies were, however, again unsuccessful with electrodes $G$ and $H$. Thus from a $4 \mu \mathrm{F}$. condenser charged and discharged 50 times/sec. (charging potential $24 \mathrm{~V}$.) through the vessel, a mean temperature rise of about $0 \cdot 25^{\circ}$ was caused without any

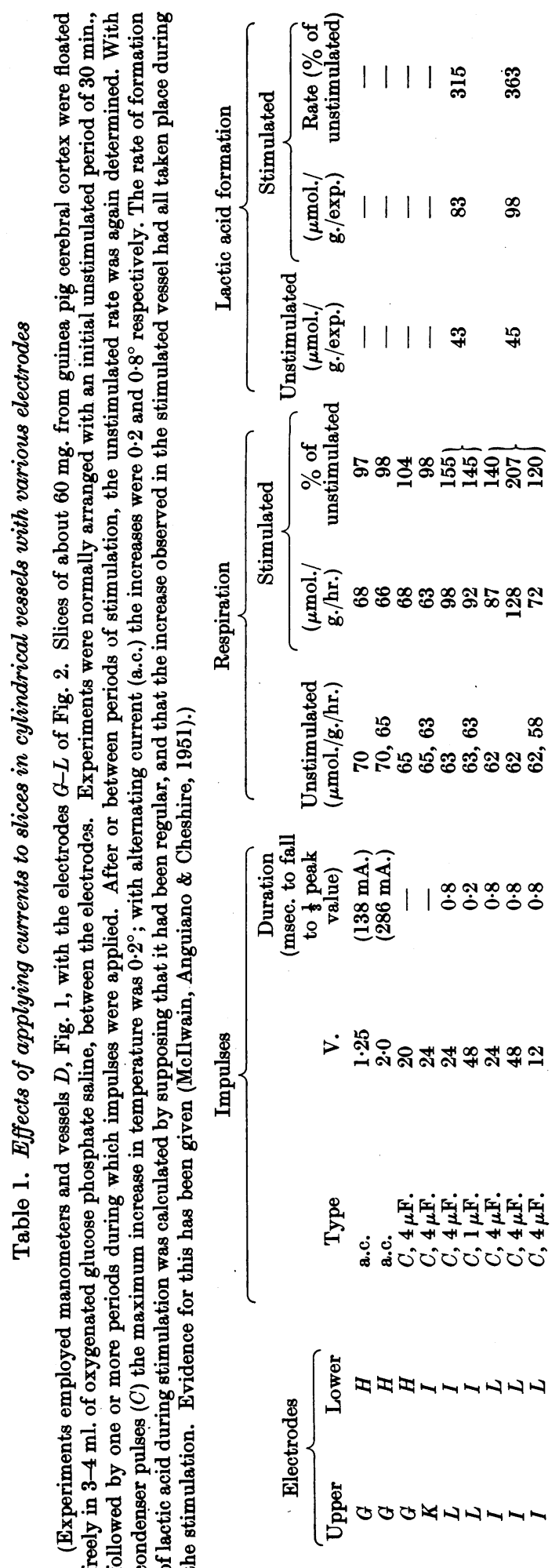


stimulation. However, the resistance of the vessel was so low that the discharge time was very brief. It was not measured accurately, but from the characteristics quoted in Table 1 it can be calculated
These observations showed that it was desirable to increase the resistance of the experimental vessel; this would decrease the energy expended by an alternating current, and increase the duration of

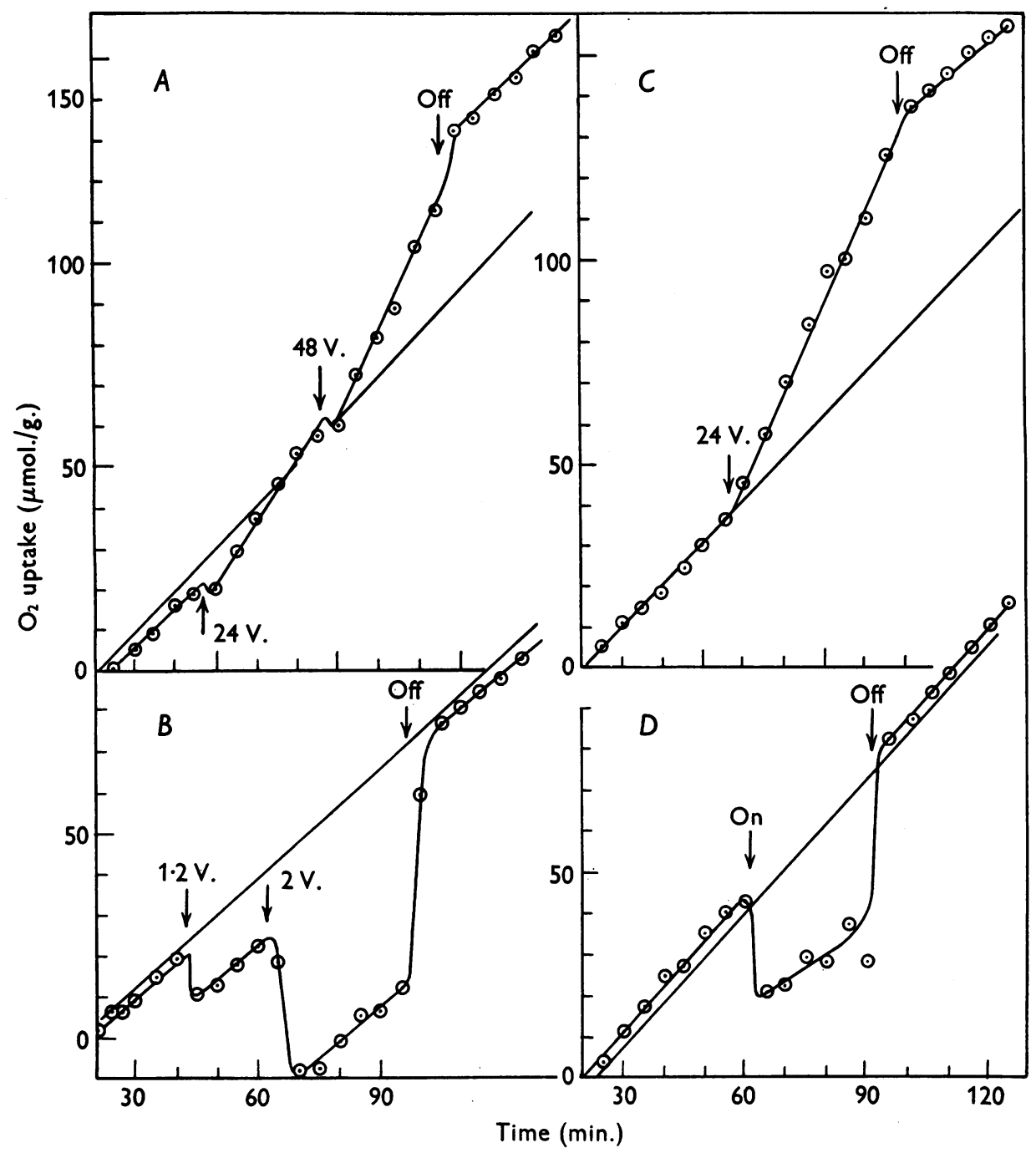

Fig. 4. Effective and ineffective impulses applied to various preparations of cerebral cortex. Experimental points are not indicated for the unstimulated control vessels. $A$, slice in cylindrical vessel, stimulated by condenser (4 $\mu \mathrm{F}$.) pulses, $100 /$ sec. at peak voltages quoted. $B$, slice in cylindrical vessel with electrodes $G$ and $H$. Alternating current, applied as indicated, had a large heating effect, but caused no change in respiratory rate. $C$, Slice fragments in conical vessel, stimulated as $A$. $D$, Slice fragments of about $3 \mathrm{mg}$. in a cylindrical vessel; current applied from a diathermy apparatus during the period indicated.

that the time taken for the applied potential to fall to one-third of its peak value was about $30 \mu \mathrm{sec}$. This is likely to be much shorter than the time needed for stimulation at minimum voltage (for references see McIlwain, 1951 $a$ ). condenser pulses. The simplest means of increasing resistance was to decrease the area of the stimulating electrodes. Electrodes $I$ to $L$ of Fig. 2 were made with this in view. Table 1 shows that the combination $K$ and $I$ was still ineffective. On the other hand, 
a simple wire end, rounded and some $1 \mathrm{~mm}$. across, was too small: evolution of gas took place from it when pulses at $24 \mathrm{~V}$. from a, $4 \mu \mathrm{F}$. condenser were applied. This did not occur when the condenser was reduced to $0.5 \mu \mathrm{F}$., but the pulses were then too brief.

The best compromise appeared to lie in relatively short lengths of wire, and the combination of $I$ and $L$ was successful. Table 1 shows that with these electrodes, large increases could be obtained in both the respiration and glycolysis of slices. The increases could be fully equal to those obtained by electrodes which made contact with the slices and also by other means (see McIlwain, 1951 a). Again, the proportional increase in glycolysis was greater than that in respiration. Threshold values, and the type of pulse most effective under the two conditions, have not been compared in detail. It is evident from Fig. 4, however, that response depends on the character of the impulses applied and is independent of their heating effects; a.c. currents were presumably ineffective because of the relatively small potential gradient or current density, and the high frequency pulses because they were too brief to excite.

\section{Stimulation of fragments of tissue}

When stimulation had been obtained without attachment of tissue to electrodes, it became possible to investigate fragments of tissue, which could be handled as suspensions. To find whether the response depended on the size of tissue-fragment, slices, prepared in the ordinary way and known to be capable of response, were cut with sharp scissors to many smaller fragments. After cutting to pieces 1-5 mg. in weight, their respiration and glycolysis remained at values very close to those in slices of 60-120 mg. Thus, the unstimulated values during the experiments of Table 2 were 65-73 $\mu \mathrm{mol}$. $\mathrm{O}_{2} /$ g. wet wt. $/ \mathrm{hr}$. and 18-25 $\mu \mathrm{mol}$. lactic acid $/ \mathrm{g} . / \mathrm{hr}$.

Pulses effective with slices were effective with fragments of slices. Table 2 quotes instances of increase in respiration to $170 \%$ and of glycolysis to $300 \%$ of the unstimulated values. Fragments of the tissue occasionally became caught and attached to one of the stimulating electrodes, but for most of the time all were suspended and no marked difference was seen to result from the occasional attachment. Further, response could be obtained in the much finer suspensions of tissue obtained by chopping the whole cerebral cortex. The making and handling of such preparations is described in the experimental part.

In the instances tried, little or no response has been given by homogenates (see Table 2). The homogenates were made (see above) and studied in the same saline as that used for the slices. This may not

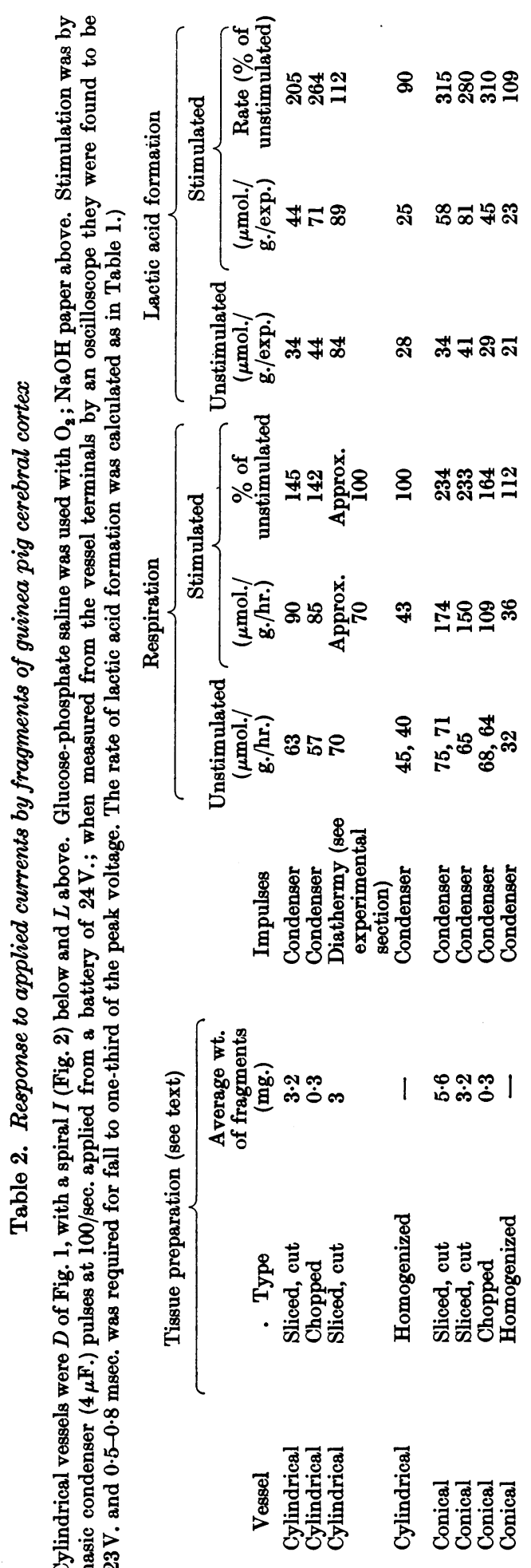


be an optimal one (see however, Elliott \& Libet, 1942; Elliott, 1948; and Cavanaugh, 1950), but we have not at present explored preparations intermediate between this and the chopped brain.
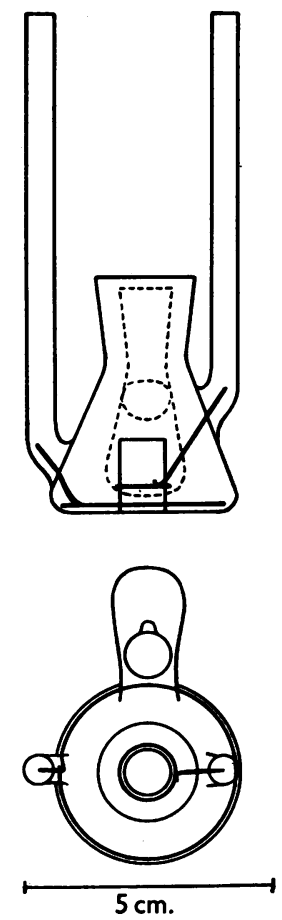

Fig. 5. Conical electrode vessels $E$. The heavier lines represent the wire electrodes.

\section{Conical vessels}

The cylindrical vessels were initially devised with the intention of placing tissue within an electric field which would be uniform at any one time. Other factors led to departure from this intention, and the most successful combination of electrodes, reached as a result of the experiments described above, had its greatest field density within a shape which approached a cone. Conical vessels have many advantages in manometric work. Agitation of fluid and surface-volume relationships are more satisfactory than in cylindrical vessels. Various electrodes have therefore been placed within ordinary conical Warburg vessels. The arrangement finally chosen was the simple one shown in Fig. 5. Here one wire is placed round the outside of the centre well towards the top of the expected fluid level, and another round the inside angle of the base. In developing this, the size of the vessel was chosen as being one commonly used. Different gauges and lengths of wire were then tried in order to arrive at those which, with a moderatesized condenser, gave vessels of such a resistance that the time of discharge would approach that known to give a good metabolic response. The size of condenser was chosen as being one which did not give too great a heating effect when charged and discharged through the saline at some 50 times/sec. (for choice of this frequency see McIlwain, 1951 $a$ ). A single turn of each wire was found preferable as giving least opportunity for entangling tissues. The sizes of wire found suitable were between 0.19 and $0.46 \mathrm{~mm}$. in diameter (36 and 26 gauge). The plating brought these sizes to about 0.21 and $0.48 \mathrm{~mm}$.

Excellent metabolic responses were obtained in these vessels with fragments of tissue (Table 2). Both respiration and glycolysis increased greatly in slices and in chopped brain cortex.

\section{Application to various parts of the brain, and to the action of drugs}

Preliminary indications are given in Table 3 of the application of the present methods. The cerebellum of many small mammals is convoluted so that if slices are cut from it in the ordinary way, fragments of a few mg. in weight are obtained. These can, however, be handled satisfactorily by present methods, and were found to respond to stimulation. Adequate preparations were also obtained from basal ganglia and from the brain stem.

\section{Table 3. Response to applied currents by different parts of the brain; action of atropine}

(Experiments employed conical vessels and diphasic condenser pulses of a peak potential of 20-23 V. and duration (at one-third max. voltage) of $0.5-0.8 \mathrm{msec}$. The tissue was as fragments of $1-4 \mathrm{mg}$. in 3.5 ml. glucose-phosphate saline with $\mathrm{Ca}$ salts. The successive periods were each of about $30 \mathrm{~min}$., beginning 20-30 min. after death of the animal; after the second, atropine was tipped in from the side arm of the vessel.)

\section{Tissue}

Guinea pig cerebral cortex Guinea pig cerebellar cortex Guinea pig basal ganglia Rat cerebellar cortex Rat brain stem
Respiration in successive periods ( $\mu \mathrm{mol} . / \mathrm{g}$. dry wt./hr.)

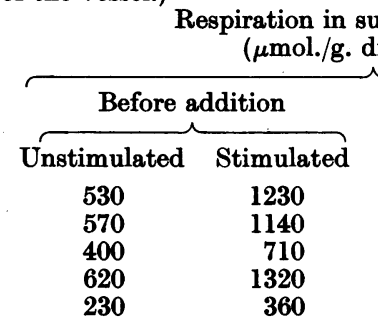

\begin{tabular}{|c|c|}
\hline \multicolumn{2}{|c|}{ With $10^{-3}$ M-atropine } \\
\hline Stimulated & Unstimulated \\
\hline 805 & 505 \\
\hline 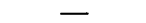 & \\
\hline $\begin{array}{l}730 \\
260\end{array}$ & $\begin{array}{l}590 \\
210\end{array}$ \\
\hline
\end{tabular}


Substances can be added to the metabolizing tissue from the side arms of the vessels without interrupting the electrical stimulation. A survey of various agents showed that some, including atropine, greatly decreased the electrically stimulated respiration and glycolysis of slices fixed to electrodes; they were, however, without action on the same processes in unstimulated slices (McIlwain, $1951 b$ ). Table 3 shows that the respiration and glycolysis of fragments of brain, unattached to electrodes, were also unaffected by atropine in the unstimulated condition, but during electrical stimulation the processes were considerably inhibited by atropine. The activity induced in the fragments is likely to be different in certain respects from that in the intact brain, but the present observations together with those previously described (McIlwain, Anguiano \& Cheshire, 1951; McIlwain, $1951 b$ ) indicate many similarities.

\section{DISCUSSION}

The only methods previously available for studying the metabolism of the central nervous system in differentstates of activity have been those employing whole animals. Perfusion has been little used. The main methods have been $(a)$ analysis of blood entering and leaving the organ, with measurement of its rate of flow ; and $(b)$ analysis of the brain itself, often after rapid fixing and dissection. Isotopic and potentiometric techniques (Davis, McCulloch \& Roseman, 1943-4 ; Gurdjian, Webster \& Stone, 1946) can be regarded as special instances of these general methods.

The present procedure may be described as an in vitro method in distinction to the previously available in vivo ones. As such it is complementary to them. It would have an important analytical function even if the other methods were easy to apply. This, however, is not the case. Thus, many of the changes which accompany different states of activity in the brain are in rates rather than in types of metabolism. The in vivo methods give metabolic rates only indirectly and after many individual analyses. With the method of fixing and analysis, the analyses are necessarily on different groups of animals fixed at different times in relation to the experimental treatment. In the case of deter. mination by arterial-venous difference, measurement of blood flow itself may need many individual analyses (Schmidt, 1950). This contrasts sharply with the present method in which direct measurements are obtained of oxygen uptake or acid formation in the same piece of tissue under different conditions, and in which very different conditions can, if necessary, be applied to pieces of material from adjacent parts of the brain.

Further, it is not easy to apply the in vivo methods, including perfusion, to small parts of the brain. To do this by arterial-venous difference would mean tapping the blood entering and leaving the particular area, and this can be done only to a very limited extent. Fixing and analysis can be applied to parts of the brain when these are readily recognizable, and when substances which are stable in the tissue are involved, but are not applicable to processes such as respiration. Also with, for example, phosphate fractions the method would require freezing and dissecting the frozen tissue in which it is especially difficult to separate the same small area from each of a series of animals.

Considering the analytical function of the present method, it is noteworthy that much of the present knowledge of whether a substrate will support the activity of the brain is derived with difficulty from experiments in vivo with hypoglycaemic animals. Agents, normal to the body or not, can be applied to the tissues in vitro with greater certainty that the agents themselves will be responsible for any observed change. The separated tissue is obviously physically altered in preparation and in an unusual environment, but many of its constituent elements need not be and can be examined under a range of electrical and chemical conditions much wider and more closely controlled than those which can be applied in a whole animal.

The present techniques can obviously be applied to tissues other than those of the brain. This study has been commenced in these laboratories (Kratzing, 1951). Smaller apparatus has also been made on the present principles, and in instances when manometric measurements are not required, very simple arrangements of two wires in a vessel would be adequate for stimulation. We have also, in working with the brain, reported largely investigations of energy-yielding and associated processes, although the present methods are applicable also to studying changes more immediately associated with the passage of nerve impulses.

The methods now developed for stimulating tissue-fragments without fixing to electrodes do not entirely replace those in which slices are held in stimulating electrodes. When sufficient coherent material is available, stimulation by contact with relatively large pieces of tissue remains most suitable for studying labile tissue constituents, for the application of defined potential gradients especially in particular directions in relation to the tissue, and for the application of certain types of impulses.

\section{SUMMARY}

1. Apparatus has been devised so that fluctuating potential gradients can be applied to portions of tissues of between 0.2 and $100 \mathrm{mg}$. in weight, while these are floating freely in oxygenated nutrient salines. 
2. Condenser pulses, of time constant about 0.5 msec., could be applied in this apparatus from condensers of about $4 \mu \mathrm{F}$. without causing any changes which might be mistaken for metabolic ones. A maximum potential gradient of about $20 \mathrm{~V} . / \mathrm{cm}$. was found to alter the metabolism of pieces of cerebral cortex which were floating in the apparatus in a balanced glucose saline. Respiration increased to up to $200 \%$ of its initial rate and lactic acid formation to some $300 \%$.
3. Similar increases were shown with cerebellar tissues and fragments from the basal ganglia and brain stem. The increases were partly inhibited by concentrations of atropine which had very little, if any, effect on the levels of respiration and glycolysis normal to the tissues.

I am greatly indebted to Mr P. Ayres and to Mr J. D. Cheshire for assistance during these experiments, and to Mr P. Ayres also for making some of the apparatus used.

\title{
REFERENCES
}

Cavanaugh, D. J. (1950). Proc. Soc. exp. Biol., N.Y., 75, 607.

Davis, E. W., McCulloch, W. S. \& Roseman, E. (1943-4). Amer. J. Psychiat. 100, 825.

Elliott, K. A. C. (1948). J. Neurophysiol. 11, 473.

Elliott, K. A. C. \& Libet, B. (1942). J. biol. Chem. 143, 227.

Gurdjian, E. S., Webster, J. E. \& Stone, W. E. (1946). Proc. Ass. Res. nerv. men. Dis. 26, 195.
Kratzing, C. C. (1951). Biochem. J. (Submitted for publication.)

McIlwain, H. (1951 a). Biochem. J. 49, 382.

McIlwain, H. (1951 b). Brit. J. Pharmacol. (in the Press).

McIlwain, H., Anguiano, G. \& Cheshire, J. D. (1951). Biochem. J. 50, 12.

Schmidt, C. F. (1950). The Cerebral Circulation in Health and Disease. Illinois: Thomas.

\section{Further Investigations on Fish Tropomyosin and Fish Nucleotropomyosin}

\author{
By G. HAMOIR \\ Laboratoire de Biologie générale, Université de Liège
}

(Received 15 May 1951)

The first method for preparing tropomyosin from muscles of several animals (rabbit, pig, horse and whiting) was described in 1948 by Bailey. Minced muscle is dehydrated with organic solvents and extracted with M-potassium chloride for $12 \mathrm{hr}$. Tropomyosin is isolated from the extract by precipitating at $\mathrm{pH} \mathrm{4.3-4.5}$, redissolving in water at neutral $\mathrm{pH}$, and fractionating with ammonium sulphate between 45 and $70 \%$. The amount of tropomyosin in rabbit muscle has been estimated by this method to be $0.47 \mathrm{~g} . / 100 \mathrm{~g}$. fresh muscle weight.

Another method of preparation has been given recently by the author for carp muscle (Hamoir, 1951). The minced muscle is submitted to two successive extractions for short periods of time (10$20 \mathrm{~min}$.) with potassium chloride-phosphate solutions: the first of pH 5.0 $(I=0.25)$, the second of pH 5.5 $(I=0.6)$ containing $0.3 \%$ sodium adenosinetriphosphate. The first extract contains the muscle globulins soluble at low ionic strength and some tropomyosin; the second myosin, nucleotropomyosin and tropomyosin. The method of isolation of nucleotropomyosin and tropomyosin from these extracts does not differ essentially from Bailey's method. Nucleotropomyosin can easily be separated from tropomyosin : it coprecipitates with the myosin at low ionic strength and neutral $\mathrm{pH}$ while tropo- myosin remains in solution. The total yield of tropomyosin and nucleotropomyosin isolated by this method from fish muscle does not amount to more than $0.1 \mathrm{~g} . / 100 \mathrm{~g}$. fresh muscle weight.

The difference in yield by the two methods has led us to try to account for the discrepancy. Fish muscle was treated following the author's method and tropomyosin was extracted from the residue by Bailey's (1948) method. The total yield of tropomyosin was then comparable with that obtained by Bailey for rabbit muscle. New investigations have also been carried out on fish nucleotropomyosin. The content of ribonucleic acid and its splitting under various conditions have been examined more closely, and the ribonucleic acid has been more fully characterized by its ultraviolet absorption in $\mathrm{N}$-perchloric acid and by quantitative estimation of the nitrogenous bases present.

\section{METHODS}

\section{Preparation of fish tropomyosin and fish nucleotropomyosin}

Carp muscle was used as starting material. Tropomyosin was prepared from the residue obtained after extracting twice by the author's method (Hamoir, 1951) and subsequently treating it according to Bailey's (1948) method. As some 